\title{
Status of the HOLMES experiment to directly measure the electron neutrino mass with a calorimetric approach.
}

Giovanni Gallucci $^{* a}$, B. Alpert ${ }^{e}$, M.Balata $^{f}$,D.T. Becker ${ }^{e}$,D.A. Bennett ${ }^{e}$, A.Bevilacqua ${ }^{a}$, M. Biasotti $^{a b}$, M. Borghesi $^{c d}$, G.Ceruti $^{d}$, M. De Gerone ${ }^{a}$, R.Dressler ${ }^{e}, \mathbf{M}$. Faverzani $^{c d}$, E. Ferri ${ }^{c d}$, J.W. Fowler ${ }^{e}$, J.D. Gard ${ }^{e}$,F.Gatti $^{a b}$, A. Giachero ${ }^{c d}$, J.P. Hays-Wehle $^{e}$, S.Heinitz ${ }^{g}$, G.C. Hilton $^{e}$, U. Koster $^{h}$, M. Lusignoli ${ }^{l}$ J.A.B. Mates ${ }^{e}$, S.Nisi $^{f}$, A. Nucciotti ${ }^{c d}$, L.Parodi ${ }^{a}$, G. Pessina ${ }^{d}$, A. Puiu ${ }^{c}$, S.Ragazzi ${ }^{c d}$, C.D. Reintsema ${ }^{e}$, D.R. Schmidt $^{e}$,D. Schuman ${ }^{g}$, F.Siccardit $^{a}$, M.Sisti $^{c d}$, D.S. Swetz ${ }^{e}$, J.N. Ullom ${ }^{e}$, L.R. Vale ${ }^{e}$ , INFN, Sezione di Genova - Genova, Italy

${ }^{b}$ Dipartimento di Fisica, Università di Genova - Genova, Italy

${ }^{c}$ Dipartimento di Fisica, Università di Milano-Bicocca - Milano, Italy

${ }^{d}$ INFN, Sezione di Milano-Bicocca - Milano, Italy

${ }^{e}$ National Institute of Standards and Technology (NIST) - Boulder, CO, USA (3)

${ }^{f}$ Laboratori Nazionali del Gran Sasso, INFN, Assergi (AQ), Italy

${ }^{g}$ Paul Scherrer Institut, Villigen, Switzerland

${ }^{h}$ Institut Laue-Langeving, Grenoble, France

${ }^{i}$ Dipartimento di Chimica, Universita' di Genova - Genova, Italy

${ }^{l}$ Istituto Nazionale di Fisica Nucleare, Sezione di Roma 1, Roma, Italy

${ }^{m}$ Multidisciplinary Centre for Astrophysics (CENTRA-IST), University of Lisbon, Lisbon, Portugal

E-mail: giovanni.gallucciege.infn.it, bradley.alpertenist.gov,

marco.balata@lngs.infn.it, dan.becker@nist.gov,

douglas.bennett@nist.gov, adriano.bevilacqua@ge.infn.it,

michele.biasotti@ge.infn.it, matteo.borghesi@mb.infn.it,

giancarlo.ceruti@mib.infn.it, Matteo.degerone@ge.infn.it,

ag.rugard.dressler@psi.ch, elena.ferri@mib.infn.it,

joe. fowler@nist.gov, johnathon.gardenist.gov,

flavio.gatti@ge.infn.it, andrea.giacchero@mib.infn.it,

jphwehle@gmail.com, stephan.heinitz@psi.ch, koester@ill.fr,

gene.hilton@nist.gov, Maurizio.Lusignoli@romal.infn.it,

john.mates@nist.gov, stefano.nisi@lngs.infn.it,

angelo.nucciotti@mib.infn.it, luigi.parodi@ge.infn.it,

Gianluigi.pessina@unimib.it, Stefano.Ragazzi@mib.infn.it,

carl.reintsema@nist.gov, dan.schmidtenist.gov,

dorothea.schumannepsi.ch, fabio.siccardiege.infn.it,

monica.sisti@unimib.it, daniel.swetz@nist.gov, joel.ullomenist.gov,

leila.vale@nist.gov 
The measurement of neutrino masses is still one of the most compelling issues in modern particle physics. HOLMES is an experiment that aims to measure the effective $v_{e}$ mass using a calorimetric approach. It will measure the spectrum end point of the electron capture (EC) decay of ${ }^{163}$ Ho. The very low Q-value $(2.8 \mathrm{keV})$ of the decay and its half life (4570 y) are optimal to reach simultaneously a reasonable activity to have sufficient statistics in the end-point, reducing the pile-up probability and have a small quantity of ${ }^{163}$ Ho embedded in the detector not to alter significantly its heat capacity. Holmium will be implanted into a micro calorimeter made by a metallic absorber coupled to transition edge sensor (TES). Each detector will be implanted with around $300 \mathrm{~Bq}$ of holmium and the goal of the experiment is implanting $\approx 500$ detectors $(8 \times 64$ array of detectors) to reach an accuracy of the order of $\mathrm{eV}$. In this contribution, we show the HOLMES experiment with its physics reach and technical challenges, along with its status and perspectives.

The 21st international workshop on neutrinos from accelerators (NuFact2019) August 26 - August 31, 2019

Daegu, Korea

* Speaker. 


\section{The HOLMES Experiment}

HOLMES is an experiment that aims to measure the effective $v_{e}$ mass using a calorimetric approach [1]. It will measure the spectrum end point of the electron capture (EC) decay of ${ }^{163} \mathrm{Ho}$. The very low Q-value (2.8 keV [2]) of the decay and its half life (4570 y) are optimal to reach simultaneously a reasonable activity to have sufficient statistics in the end-point, reducing the pileup probability and have a small quantity of ${ }^{163} \mathrm{Ho}$ embedded in the detector not to alter significantly its heat capacity. Holmium will be implanted into a micro calorimeter made by a metallic absorber coupled to transition edge sensor (TES). Each detector will be implanted with around $300 \mathrm{~Bq}$ of holmium and the goal of the experiment is implanting $\approx 500$ detectors (8x64 array of detectors) to reach an accuracy of the order of $\mathrm{eV}$. In the calorimetric approach, the source is embedded inside the detector and the energy released in the decay process is entirely contained, except for the fraction taken away by the neutrino. Both the issues related to the use of an external source and the systematic uncertainties arising from decays on excited final states are eliminated. The released energy will be measured using 16 sub-arrays of Transition Edge Sensor based microcalorimeters (64 TES for each array) with ${ }^{163}$ Ho source (EC) embedded inside. Each TES will have an energy resolution of $\approx 1 \mathrm{eV} \mathrm{FWHM,} \mathrm{a} \mathrm{time} \mathrm{resolution} \mathrm{of} \mathrm{about} 1 \mu \mathrm{s}$ and will be read out using microwave multiplexed rf-SQUIDs in combination with a ROACH2 based digital acquisition system. An activity of $300 \mathrm{~Bq}$ will be implanted in each micro-calorimeter allowing to collect about $3 \times 10^{13}$ decays. HOLMES will be an important step for a calorimetric approach as an alternative to spectrometry and will also establish the potential of this approach to extend the sensitivity down to $0.1 \mathrm{eV}$ and lower. In the next chapters we will present the status of the HOLMES in particular the activities concerning the ${ }^{163} \mathrm{Ho}$ isotope production, purification and implantation (sections 2, 3 and 4), the TES pixel design and optimization and the multiplexed array read-out characterization (section 5).

\section{Holmium production and distillation}

The ${ }^{163} \mathrm{Ho}$ is produced with neutron irradiation of ${ }^{162} \mathrm{Er}$ [3]. About $700 \mathrm{mg}$ of erbium are already irradiated at the Institut Laue-Langevin (ILL, Grenoble, France) producing around 150 $\mathrm{MBq}$ of ${ }^{163} \mathrm{Ho}$. The presence in the sample of other erbium isotopes and rare earth (holmium and dysprosium) contaminations creates other radioactive isotopes. Embedding those isotopes could deteriorate the sensitivity of neutrino mass measurements increasing the pileup or changing the detector heat capacity. Two different procedures will be implemented to purify the sample.A chemical purification to remove all non-holmium isotope that is done at the Paul Scherrer Institute (PSI, Villigen Switzerland) [4]. An isotopic separation crucial to remove the ${ }^{166 m} \mathrm{Ho}$, produced during the neutron irradiation. This isotope is a beta emitter with half life of about $1200 \mathrm{y}$ and induces background below $5 \mathrm{keV}$. In order to do this an implanter (section 4 ) will be used. After the chemical purification, the purified Ho sample is sent to the Physics Department of Genova University in oxide form $\left(\mathrm{Ho}_{2} \mathrm{O}_{3}\right)$. To avoid chemical shifts of the end-point, only holmium in the metallic chemical form must embedded in the detector so a reduction-distillation (RD) procedure will be performed to remove oxygen from oxide. A dedicated evaporation chamber has been developed to perform the RD procedure and the whole procedure has an efficiency of about $73 \%$ [5]. 


\section{Sputter target production}

The sputter ion source of the implanter needs a metallic cathode containing the ${ }^{163} \mathrm{Ho}$. The $\mathrm{RD}$ procedure produces holmium powder so we decided to realize a sintered sputter target mixing the Ho with fine grained powder $(\leq 40 \mu \mathrm{m})$ of $\mathrm{Ti}, \mathrm{Ni}$ and $\mathrm{Sn}$. Ti and $\mathrm{Ni}$ have been chosen because they create intermetallic compounds with holmium and increase the mechanical stability of the target. Sn has been chosen to decrease the melting temperature of the compounds. The mix is placed is a copper structure, pressed at $350 \frac{\mathrm{bar}}{\mathrm{cm}^{2}}$ and heated at $850{ }^{\circ} \mathrm{C}$ in low vacuum environment (about $10^{-2}$ mbar) for 4 days. A first target made with non radioactive ${ }^{165} \mathrm{Ho}$ is ready.The crystallographic measurements and SEM-EDS analysis show two different phases: a $\mathrm{Ti}_{2} \mathrm{Ni}_{2} \mathrm{Sn}$ matrix with homogeneously distributed islands of HoNiSn. This configuration is suitable for HOLMES purposes.

\section{Holmium implantation}

The HOLMES implanter has been produced by DANFYSIK and consists of six different components (fig.1). The main component is a sputter ion source[6] made by a small vacuum chamber where a flux of argon is ionized via an electron current produced by a filament (thermionic effect). The electron current is adjustable tuning the filament current and the anode voltage. A sputter target is mounted in the chamber and could reach voltage up to $600 \mathrm{~V}$ : the argon ions hit the target and sputter the material that is subsequently ionized in the source. An acceleration section is attached to the ion source and have an exit aperture of about $1 \mathrm{~cm}^{2}$. The ionized sputtered materials could be accelerated until a maximum energy of $50 \mathrm{keV}$. The third section is a magnetic mass analyzer. It consists of a magnetic dipole with a bending radius of $46 \mathrm{~cm}$ and capable to reach a field intensity up to $1.1 \mathrm{~T}$. The fourth and fifth components are a focusing electrostatic triplet, to refocus the beam and a magnetic XY scanning stage, to measure the beam spot. At the end of the implanter the last section is a vacuum chamber. In this chamber the detector array will be hit by the holmium beam and a smaller argon sputter source will allow a simultaneous gold evaporation to control the

${ }^{163} \mathrm{Ho}$ concentration and to deposit a final gold layer to prevent the oxidation [7]. The vacuum level inside the implanter is less than $10^{-6}$ mbar and the beam spot is around $4.5 \mathrm{~mm}$ FWHM in the focal point. Here there are a set of slits to cut the tails of the beam, followed by a Faraday cup to measure the beam current intensity. The current of the beam reaches intensity from 0.7 to $15 \mathrm{~mA}$ at $30 \mathrm{keV}$, dependent from materials (table from producer). In our configuration the ${ }^{163} \mathrm{Ho}$ current will reasonably depend from the fraction of isotope in the sputter target (less than 1\%) and it will be expected around $10-100 \mu \mathrm{A}$. The whole system provides a separation between ${ }^{163} \mathrm{Ho}$ and ${ }^{166 m} \mathrm{Ho}$ better than $10^{5}$ (simulation from producer). The first three components are installed and are being set up in INFN laboratories at Genoa University. The target chamber has been tested at Milano Bicocca University and the focusing triplet and the scanning stage are not installed yet. The integration is expected in 2020. In the first test phase the implanter will be tuned on ${ }^{165} \mathrm{Ho}$ and the beam current and spot will be verified.

\section{Operation and readout}

The HOLMES detectors are micro calorimeter made of a $2 \mu$ m-thick gold absorber coupled 


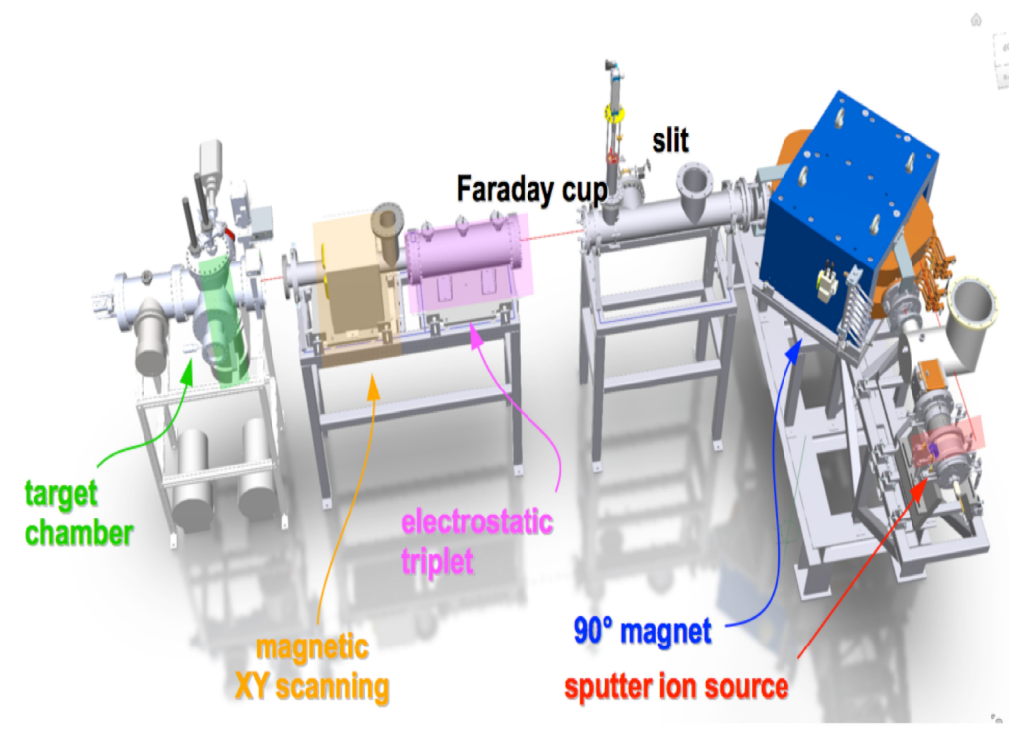

Figure 1: The scheme of the implanter.

with a Molybdenum/copper TES suspended on $\mathrm{Si}_{2} \mathrm{~N}_{3}$ membrane. Four different designs have been tested to reach the best performance in terms of energy and time resolution, pulse duration and full energy containment. The HOLMES detectors are read out with the microwave multiplexing system ( $\mu$ mux), which is based on the use of the rf-SQUIDs. The acquisition is done using a ROACH-2 acquisition board. The TES design, production and preliminary test is done in collaboration with @ NIST. The final configuration has been tested in a dilution cryostat capable to reach about $60 \mathrm{mK}$ [8]. The time response is determined by the working resistance of the TES and by the inductance of the bias circuit. A stray inductance is to be added in order to tune the time response to the desired $10 \mu$ s rise time. In order to successfully apply the pileup resolving algorithms that allow to push the time resolution down to $1 \mu \mathrm{s}$, a $500 \mathrm{kHz}$ sampling rate of each pulse is needed. This constraints, combined with the total ADC bandwidth, result in a multiplexing factor of $\approx 30$ per each ROACH-2 acquisition board. Finally, we tested the energy resolution of the detectors using an X-ray emitting source composed of $\mathrm{Al}(1.48 \mathrm{keV}), \mathrm{Cl}(2.62 \mathrm{keV}), \mathrm{Ca}(3.63 \mathrm{keV})$ and $\mathrm{Mn}(5.98$ $\mathrm{keV}$ fig.2) obtaining an energy resolution of $4.5 \mathrm{eV}$ at $2.8 \mathrm{keV}$.

\section{Conclusions}

Three different batches of ${ }^{163} \mathrm{Ho}$ have been produced, purified and moved to INFN laboratories in Genova. The procedures to distillate holmium and to fabricate the sputter target are tested. Some refinements are needed. The installation of the main parts of the implanter is mainly finished and the integration of the remaining parts are expected in 2020. HOLMES detectors production procedure is defined and the firsts (not implanted) detectors are being characterized. Readout is on test and is almost ready. With 32 pixel a sensitivity of $\approx 10 \mathrm{eV}$ is expected. With the full sample of setectors a sensisivity of $\approx 1.5 \mathrm{eV}$ is expected in three years of data taking. The first test are expected in 2020 


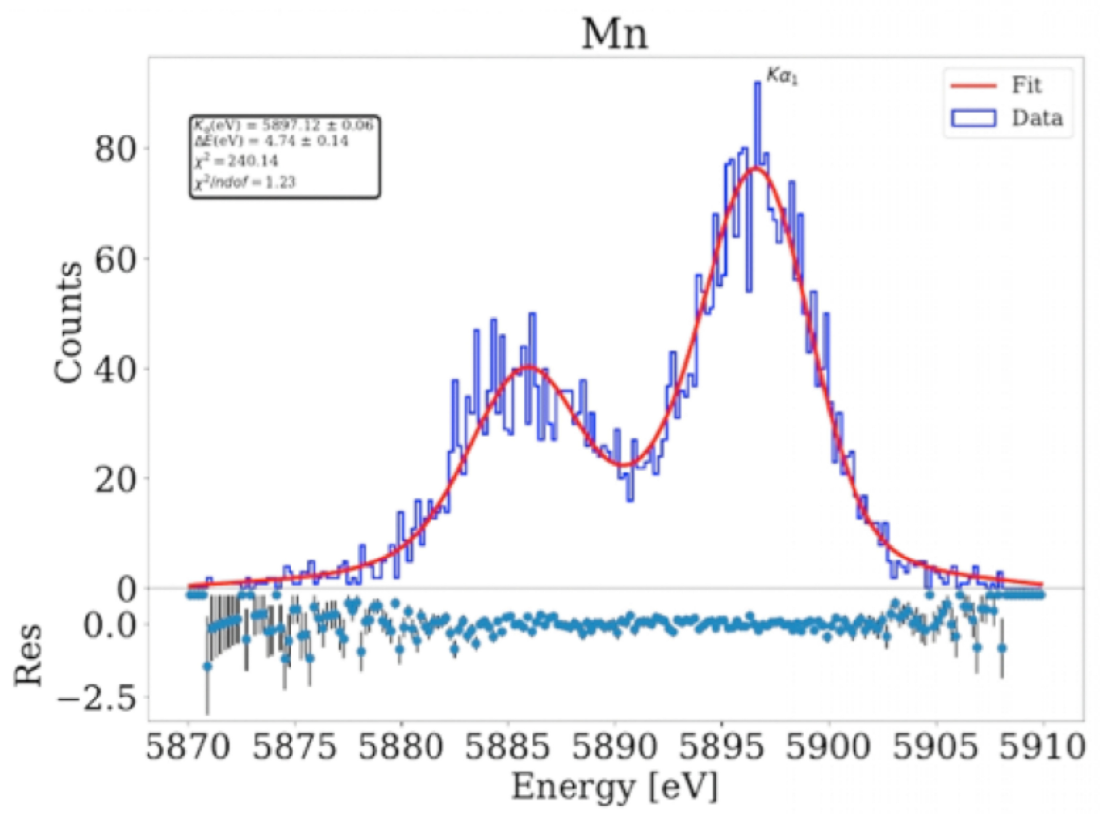

Figure 2: Measurement of X-ray from a manganese source .

Acknowledgements - The HOLMES experiment is funded by the European Research Council under the European Unions Seventh Framework Programme (FP7/2007-2013)/ERC Grant Agreement No. 340321.

\section{References}

[1] B.Alpert, et al., HOLMES - The Electron Capture Decay of ${ }^{163}$ Ho to Measure the Electron Neutrino Mass with sub-eV sensitivityThe European Physical Journal C 75112 (2015)

[2] S.Eliseev et al., Direct Measurement of the Mass Difference of ${ }^{163} \mathrm{Ho}$ and ${ }^{163} \mathrm{Dy}$ Solves the Q-Value Puzzle for the Neutrino Mass Determination,Phys.Rev.Lett. 115 (2015) no.6, 062501

[3] Engle et al.,Evaluation of 163 Ho production options for neutrino mass measurements with microcalorimeter detectors, Nucl.Instrum.Methods B 311 (C) 131138 (2013)

[4] S.Heinitz et al., Production and separation of 163Ho for nuclear physics experiments, PLoS One 13 (2018) no.8, e0200910

[5] G.Gallucci et al., ${ }^{163}$ Ho Distillation and Implantation for HOLMES Experiment,J.Low.Temp.Phys. 194 (2019) no.5-6, 453-459

[6] Z.Nouri et al., A Penning sputter ion source with very low energy spread, Nucl.Instrum.Methods A 614, (2010) 174

[7] A. Orlando et al., Microfabrication of Transition-Edge Sensor Arrays of Microcalorimeters with ${ }^{163} \mathrm{Ho}$ for Direct Neutrino Mass Measurements with HOLMES,J.Low.Temp.Phys. 193 (2018) no.5-6, 771-776

[8] B.Alpert et al.,High-resolution high-speed microwave-multiplexed low temperature microcalorimeters for the HOLMES experiment,Eur.Phys.J. C79 (2019) no.4, 304 